I RECENSIÓN

\title{
Masferrer, Aniceto y Emilio García-Sánchez (eds.) Human Dignity of the Vulnerable in the Age of Rights (Suiza: Springer, 2016)
}

\author{
Alejandro Salcedo Romo \\ Universidad Panamericana \\ México
}

Fecha de recepción: 01/12/2016 I De publicación: 19/12/2016

"Este volumen está enfocado en explorar un tema que, en superficie, podría parecer solamente como un tópico de moda. De hecho, es mucho más que una tendencia o tópico de moda. Se relaciona con un asunto antiguo y permanente que está íntimamente relacionado con la vida de las personas y sus necesidades básicas: el reconocimiento y la protección de la dignidad individual, en particular la dignidad inherente de los seres humanos más vulnerables." (p. V) Con esta frase se apertura el libro de "Human Dignity of the vulnerable in the age of rights", el cual pertenece a la colección Ius Gentium de la prestigiosa editorial Springer.

El tema es perfectamente delimitado y se mantiene fiel hasta la última página: la dignidad humana de los vulnerables en la era de los derechos. Así, la vulnerabilidad se nos presenta como parte inherente de la condición humana, y por lo tanto como un llamado a que el derecho reconozca y proteja los derechos de todos los individuos "particularmente de aquellos que pueden aparecer como más vulnerables $\mathrm{y}$ frágiles." (p.V), algo similar al trabajo realizado recientemente (1999) por Alasdair MacIntyre en el ámbito de la filosofía moral y política ${ }^{1}$, aunque en este volumen se extiende y profundiza desde un perspectiva multidisciplinar.

La obra que tenemos en frente responde a una paradoja que suele pasar desapercibida, y es que en la época en que los derechos humanos han adquirido su mayor auge consolidándose en declaraciones e instituciones de ámbito nacional e internacional, se ha vulnerado de manera constante -'legitimándose' bajo la misma bandera de derechos humanos- a aquellos miembros más frágiles de la familia humana: no nacidos, ancianos, enfermos mentales, discapacitados, etcétera. Esta es la paradoja que es evidenciada en el libro y a la que los autores que se unen en torno a la dignidad y los derechos de los más vulnerables intentan responder

\footnotetext{
${ }^{1}$ MacIntyre, Alasdair C. Animales racionales y dependientes: por qué los seres humanos necesitamos las virtudes. 2001.
} 
constituyendo una reflexión interdisciplinar, que se materializa en cada uno de los catorce capítulos, y que queda distribuida en las tres partes que componen la obra: "Human Dignity of the Vulnerable: Ethical and Anthropological Perspectives"; "Human Dignity of the Vulnerable: Biomedical and Sociological Perspectives" y "Human Dignity of the Vulnerable in the Age of Rights: Historical, Legal Philosophical and Political Perspectives" a las que se hace alusión a continuación.

El Capítulo 1 'Vulnerability and Human Dignity in the Age of Rights' de Aniceto Masferrer y Emilio García-Sánchez -editores del volumen- se encuentra dividido en tres partes: la primera en la que se presenta la necesidad del reconocimiento de la vulnerabilidad humana como condición de respeto de la dignidad humana; la segunda que profundiza en el vínculo entre derechos humanos, dignidad humana $\mathrm{y}$ vulnerabilidad humana; y, finalmente se despliega la intencionalidad y el itinerario que seguirá la obra en su totalidad, siendo este apartado la mejor recensión a la que se puede acudir para comprender los contenidos del libro, lo que evidencia el trabajo artesanal de los editores y el reto que enfrenta la presente reseña.

Parte I 'Human Dignity of the Vulnerable: Ethical and Anthropological Perspectives' -conformado por cuatro capítulos- comienza con la intervención de Alfredo Marcos 'Vulnerability as a Part of Human Nature' que se ocupa del concepto de naturaleza humana, tema que ha vuelto al centro de los debates filosóficos contemporáneos. El autor no solamente defiende la existencia de la naturaleza humana, sino que afirma que la vulnerabilidad constituye parte esencial de ésta, postulado que confrontará a tres posturas: la de aquellos autores que niegan la existencia de la naturaleza humana, la de aquellos que la naturalizan -o la reducen a la condición animal- y la de aquellos autores que propugnan por una intervención radical de la técnica en los seres humanos -trans y post-humanistas-Para responder a estas posturas el autor hace una relectura de la filosofía aristotélica con la intención de desarrollar los tres conceptos con los que el estagirita definió al ser humano: zoon politikon logon. El desarrollo de estos términos le permite afirmar "we do have a proper place, a seat, or even more than one: the natural environment, the social world and the sphere of the spiritual. And we do have certain characteristics-let's say talents-, by nature: vulnerability, dependency and autonomy." (p. 33). Con esta afirmación Alfredo Marcos establece el vínculo con el último apartado de su capítulo, en el que presenta su reflexión en torno a la definición e implicaciones de la vulnerabilidad, la importancia que tiene en nuestro tiempo - 
distinguiendo los diversos tipos de vulnerabilidad por las que somos afectados- y finalmente, las diversas estrategias que se pueden tomar frente a la vulnerabilidad: resignación, deshumanización, reconocimiento y mitigación. Evidentemente el autor se decantará por la última opción, afirmando "The recognition of vulnerability provides us with an irreplaceable moral teaching: it teaches us the importance of solidarity and altruism. Together, social relations, mutuality, solidarity and altruism, as well as legal frameworks that promote justice, save us from extreme vulnerability.” (pp. 42-43)

En el tercer capítulo 'Ethics of Vulnerability' Adela Cortina y Jesús Conill muestran la necesidad de una reflexión cauta sobre la dimensión vulnerable de la vida, evidenciando la gran concentración que ha puesto la tradición filosófica occidental en la autonomía al momento de plantearse los proyectos de vida buena, y la relegación de la vulnerabilidad a segundo plano, a pesar de que esta constituye parte de nuestra naturaleza. Al respecto recuerdan lo establecido por autores como Williams, Nagel, MacIntyre y Nussbaum, prestando especial atención a estos dos últimos. La vulnerabilidad se presenta por lo tanto "as an essential key to design good life projects and to propose demands of justice." (p. 52), pero ¿se deriva alguna obligación moral del hecho de que los seres humanos seamos vulnerables? Esta pregunta conduce a la última parte de la intervención de
Cortina y Conill donde presentan las tres respuestas más relevantes a este respecto en la actualidad: la ética del cuidado, la ética de la responsabilidad y la ética de la cordialidad.

En el capítulo 4 'Vulnerable: To Be Between Life and Death' Aquilino Cayuela profundiza en los aspectos metafísicos de la vulnerabilidad, partiendo de afirmar "The place of every human being is between life and death [...] includes not only the temporality of the existence (like pathos) or the biography, but also the space (extension) of the corporal presence (as in flesh or incarnare) or the biology." (p. 63) Posteriormente A. Cayuela desarrollará las implicaciones que se encuentran en la afirmación del ser humano como ser entre la vida y la muerte, a partir de tres dimensiones: pathos, in carnis, y ethos. A este respecto centrará su atención en el concepto de pathos -especialmente en Aristóteles- para vincular su exposición con el término de vulnerabilidad y la necesidad del desarrollo de una path-ética, al respecto de la cual afirmará "Our path-ethical proposal aims at completing the ethical field, which is the characteristic of the ethos, like rational selfcontrol, with its specifically human elements that concur in the biography of every man, in his being between life and death. This being in the time situates us as vulnerable." (p. 70) Para el autor esta visión -que analiza el carácter paradójico del pathos en el ethos- permite 
retomar el camino a una filosofía moral realista y a una comprensión más amplia de la categoría de humanidad, misma que hace frente tanto a la concepción bidimensional de la antropología -que centra la atención o en la presencia espacial o en una ética cerrada en la comprensión racionalcomo a la visión metafísica de Sartre. En consecuencia el autor concluye proponiendo la recuperación de la metafísica desde una perspectiva más humilde, pero igualmente comprometida con el cuestionamiento sobre el Ser.

En el capítulo 5 'The Vulnerability of Life in the Philosophy of Hans Jonas' Paolo Becchi y Roberto Franzini Tibaldeo ofrecen un análisis sobre el concepto de vulnerabilidad en la filosofía de H. Jonas, comenzando con la ponderación sobre el desarrollo tecnológico de nuestros días en la filosofía de dicho autor, a través de su obra más representativa Das Prinzip Verantwortung, evidenciando como las promesas de la tecnología moderna se han convertido en amenaza. A este respecto resaltan la peculiaridad de la visión del autor estudiado que -en contraste con autores como Jaspers y G. Anders- considera que lo verdaderamente amenazador de la tecnología post Segunda Guerra Mundial es que los resultados problemáticos surgen precisamente por su empleo 'ordinario' de forma masiva. Para continuar con el desarrollo de estas ideas, Becchi y Franzini contrastan el uso e impacto de la tecnología en los tiempos pre-modernos con el uso e impacto de ésta en la actualidad, señalando que el desarrollo técnico contemporáneo ha puesto de relieve la vulnerabilidad de la naturaleza a través de los efectos generados por la intervención técnica del hombre, lo que permite afirmar -siguiendo a Jonas- que la naturaleza de nuestra acción ha cambiado de facto, haciéndonos responsables de la biósfera entera del planeta, debido al poder que ejercemos sobre ella. Evidentemente las peculiaridades del uso de la técnica en la modernidad exige nuevas consideraciones éticas ¿cómo se podría prevenir que dañemos la naturaleza en su totalidad? Y en cualquier caso ¿por qué debería el ser humano tener como objetivo preservar la existencia y la integridad de la naturaleza? ¿solamente porque vivimos en ella, y la destrucción de la biósfera sería dañina para nosotros? ¿o hay alguna base para afirmar que la preservación de la naturaleza es algo bueno en si? Los autores desarrollan la respuesta de Hans Jonas, la cual toma las dos perspectivas y les añade la dimensión de vulnerabilidad, que a través de su doble función -histórica y filosóficapermite enfatizar la compleja paradoja del desarrollo tecnológico, a la que Jonas propone hacer frente a través de la heurística del miedo. Partiendo de lo anterior Becchi y Franzini trazan un itinerario que recorre la esencial vulnerabilidad de la vida orgánica -que constituye la base para comprender la ética de H. Jonas-, la relevancia 
metafísica de la vulnerabilidad y finalmente la relevancia ética de la vulnerabilidad. Los autores nos aportan, por lo tanto, un recorrido detallado en los conceptos de la filosofía de Jonas, al igual que una reflexión en torno a la vigencia de su pensamiento particularmente al recordarnos, en una época de derechos, la importancia de nuestros deberes a través de la esencial vulnerabilidad de la vida, proponiendo convertirnos en cuidadores activos y responsables de la vulnerabilidad y de la integridad que se encuentra frente a nosotros.

La II Parte 'Human Dignity of the Vulnerable: Biomedical and Sociological Perspectives' comienza con dos capítulos a cargo de Luis Echarte, mismos que se encuentran estrechamente relacionados por dos tópicos eje: los avances de la neurociencia y la neurotecnología, y la forma en la que estos avances afectan en la comprensión de nosotros mismos, generando las denominadas Experiencias Inauténticas -IE a partir de ahora, aludiendo a sus siglas en inglés-. En el Capítulo 6 'Biotechnologies Inside the Self: New Challenges in Clinical Ontology' Echarte expone la introducción a la problemática de las IE -efectos adversos relevantes en la revolución de la neurociencia-, así como los objetivos y alcances de la Ontología Clínica. Para ello realiza una exposición a cerca de algunos tratamientos psicofarmacológicos y de la Estimulación de Cerebro Profundo -DBS, por sus siglas en inglés-
, resaltando que gracias al resultado de estudios recientes es posible encontrar en ambos métodos síntomas similares relacionados con la auto comprensión de los pacientes sujetos a estos métodos. La diferencia entre la aparición de los síntomas radicaría principalmente en el tiempo, debido a que la DBS produce los cambios asociados a dichos síntomas de forma más repentina y por lo tanto dramática. El autor prosigue identificando cuatro tipos de IE, a saber: las relacionadas con la imagen propia, las relacionadas con la espontaneidad, las de la experiencia propia de la libertad con relación al cuerpo y aquellas que afectan la visión a cerca de la humanidad. Posteriormente propone la Ontología Clínica como medio para hacer frente a las EI, identificando tanto los retos que ésta enfrenta, como los requisitos con los que se debe cumplir para ser elegido en una terapia de ontología clínica. En el último apartado Echarte realiza un recorrido que -aunque hecho por superficie- señala con agudeza la similitud de los efectos negativos que ha generado la revolución de la neurociencia -Experiencias Inauténticasrespecto a otros fenómenos contemporáneos como es el 'mejoramiento humano' o la realidad virtual.

$$
\text { En el capítulo } 7 \text { 'Paradoxes of }
$$
Authenticity: A Neuroscientific Approach to Personal Identity' el autor continúa con lo expuesto en el capítulo 6, aunque poniendo 
énfasis en las bases biológicas, sociales e intelectuales de las IE -Experiencias Inauténticas-, empleando el marco de los tres niveles del yo de Antonio Damasio. A través de estos tres niveles del yo, Echarte clarifica aspectos fundamentales de la dinámica entre las identidades prácticas y las identidades morales, al respecto de las cuales muestra como suelen involucrar creencias que no resultan ser siempre conscientes y coherentes. El trabajo de Echarte profundiza más al respecto proponiendo un modelo que evidencia la coexistencia en el mismo nivel -dislocación horizontal-o en diferente nivel -dislocación vertical- de ideas normativas contradictorias. Paralelamente el autor demuestra la influencia que tiene la tecnología y el contexto social en las creencias humanas, al igual que la subestimación que se suele dar a la influencia que estas dos tienen en el propio sistema de valores. Precisamente esta ingenuidad -la subestimación de la influencia de la sociedad y la tecnología- es la que el autor considera la mayor causa de IE en la Cultura Occidental, así como la causante de un especial tipo de vulnerabilidad: una identidad frágil, carente de la experiencia de control.

El capítulo 8 'Vulnerability at the End of Life: A Medical Perspective' a cargo de Joaquim Bosch-Barrera y Juan Vidal Bota parten de una premisa evidente e insalvable, aunque muchas veces dejada de lado "Despite recent, significant improvements in medicine, all human beings will eventually die.” (p. 167) Siendo así los autores se centran en la etapa del final de la vida, misma que siendo un claro ejemplo de la vulnerabilidad humana, debería constituirse en uno de los mayores asuntos de salud pública, ya que siendo la última etapa de nuestra vida no admite segundas oportunidades para enfrentarla de buena manera, pero ¿qué significa buena muerte? Y ¿morir con dignidad? La estructura del capítulo oscila en torno a estas preguntas, exponiendo algunas de las respuestas centrales en torno al tema: desde el momento en que se aproxima la muerte, la importancia del buen diagnóstico y el reto que supone para los médicos equilibrar la esperanza con metas realistas y alcanzables del tratamiento; la autonomía del paciente, misma que en esta etapa suele verse mermada por la dificultad que supone comunicarse o por la imposibilidad de hacerlo, por lo que los autores se detienen en torno al tema de voluntad anticipada; el control del dolor y otros síntomas, donde recuerdan que el sufrimiento no solamente tiene una dimensión física sino que además tiene una dimensión existencial; lo anterior conduce al aspecto en torno a la asistencia espiritual que deben recibir los pacientes, y los familiares, en esta etapa final; la capacidad de decidir el lugar para morir, capacidad que se ve obstruida muchas veces por la falta de acceso a cuidados paliativos en casa; finalmente Bosch-Barrera y Vidal Bota centran su atención en la importancia que tiene el 
ser capaz de detenerse cuando llega el momento de irse, reflexionando sobre temas polémicos en torno a esto, como lo son el encarnizamiento terapéutico y la eutanasia. Los autores concluyen haciendo una acertada cita de Elisabeth KüblerRoss "In this rushed and busy and overspecialized world it is more important than ever that we train physicians to care for the total needs of the patient and his family and this requires a training not only in the science of medicine but also in the art of medicine, which has something to do with a little bit more humanity." (p. 183)

En el capítulo 9 'Cosmetic Vulnerability: The New Face of Human Frailty' Emilio GarcíaSánchez centra su atención en un nuevo grupo de personas vulnerables surgido en la posmodernidad... los cosméticamente vulnerables. Aunque bien la búsqueda de la belleza se encuentra inserta en la historia, nunca había alcanzado los niveles actuales, mismos que se ven reflejados en la aparición de una multiplicidad de profesiones enfocadas al embellecimiento del cuerpo. El autor comienza por exponer los paradigmas que sostienen el modelo desenfrenado por conseguir la belleza, siendo el primer paradigma el relacionado con la liberalización y autonomía corporal, algo que podría resumirse en la expresión "My body is mine and I decide how to use it" (p. 193) expresión que deja ver la alienación del cuerpo, la capacidad de intercambiarlo dependiendo mi gusto. Estando centrada la liberalización del cuerpo en uno de los principios paradigmáticos de la medicina, el principio de autonomía, el problema aumenta ya que el único límite posible a las intervenciones estéticas radica en la autonomía del paciente. El segundo paradigma se encuentra en la creación de un ícono global de belleza, que el autor caracteriza de forma creativa como "Young, Sexy and White Bodies" (p.195) y que es el modelo que se presenta a través de los famosos en los diversos medios de comunicación. A través de estos paradigmas se invierten los valores respecto a la comprensión de la imagen del hombre, misma que normalmente se compone de vulnerabilidad, de debilidad, y de defectos incluso de carácter estético, poniendo en su lugar la aspiración -por naturaleza inalcanzable- de cuerpos perfectos, 'indestructibles', y que representan la eterna juventud. Lo anterior junto con el impacto de la cosmética ha originado -de acuerdo a García-Sánchez- la manifestación de un nuevo grado de vulnerabilidad humana, la vulnerabilidad cosmética o estética, que describe en los siguientes términos "The cosmetically vulnerable are a social group that, due to pressure and aesthetic seduction, experience a strong need to match the beauty of the fashion icon. For them it is a source of suffering if they do not reach this standard of success." (p. 202) Posteriormente expone las diversas manifestaciones de esta vulnerabilidad: vulnerabilidad por sobrepeso y 
obesidad; etnicidades cosméticamente vulnerables; grupos de edades cosméticamente vulnerables $y$ finalmente la vulnerabilidad ocasionada por el embarazo y el postparto. Al respecto de esta última resulta llamativo ver como la cultura postmoderna asocia el tener hijos con la fealdad, con la disminución de la belleza, el decrecimiento de calidad de vida y de éxito profesional. García Sánchez afirma a manera de conclusión la importancia de identificar la medicina cosmética como parte de esta nueva cara de la fragilidad humana y no como la solución, proponiendo retomar el valor ontológico de la dignidad humana el cual no cambia dependiendo de las variaciones estéticas.

La III Parte 'Human Dignity of the Vulnerable in the Age of Rights: Historical, Legal Philosophical and Political Perspectives' se encuentra conformada por cinco capítulos, comenzando con la reflexión histórica a cargo de Aniceto Masferrer y que lleva el título 'Taking Human Dignity More Humanely: A Historical Contribution to the Ethical Foundations of Constitutional Democracy' El objetivo del autor es argumentar como el concepto kantiano de autonomía ha sido mal interpretado, resaltando la continuidad histórica del pensamiento de Kant con autores previos que ya habían vinculado el concepto de derechos naturales -derechos humanos- con la dignidad de la naturaleza humana. Lo anterior lo realiza a través de un recorrido histórico que comienza por el escenarios post Segunda Guerra Mundial como el momento en el que emergieron los derechos humanos y la dignidad humana en el Derecho Internacional Público y en el Derecho Constitucional. Habiendo expuesto este panorama, el autor continúa con el resurgimiento del concepto kantiano de dignidad humana en la posguerra, especialmente en lo referente a la exaltación de la autonomía. El análisis del autor al respecto de este apartado lo hace partiendo de afirmar como el concepto kantiano de dignidad humana ha sido malinterpretado dado la exaltación de la autonomía, para ello presentará el pensamiento de autores que del siglo XVII y XVIII y que utilizaron el término de dignidad humana antes que Kant. El itinerario histórico propuesto, acompañado de las reflexiones de gran lucidez del autor, permite completar el objetivo de fondo del artículo: la afirmación de que para poder defender la dignidad humana en esta época caracterizada por los derechos, es necesario retomar el pensamiento clásico, en tanto que éste tenía claro que el fundamento de la dignidad y los derechos humanos se encuentra en la naturaleza humana, por lo que resulta impostergable para la filosofía contemporánea -especialmente para la iusfilosofia- retomar y ahondar en torno a esta cuestión.

En el capítulo 11 Roberto Andorno nos propone desde su título- una pregunta que se antoja 
ineludible para el libro que nos ocupa ¿Es la vulnerabilidad el fundamento de los Derechos Humanos? Para dar respuesta a ello el autor comienza por describir las diversas formas en las que ha sido considerada la vulnerabilidad en los discursos contemporáneos éticos y jurídicos, lo que ha ocasionado una vinculación estrecha entre el primero y el último, haciendo que la reflexión en torno a esta realidad desde el discurso ético en el que Andorno destaca el trabajo de Levinas y Ricour- ha influenciado benéficamente en el desarrollo de la teoría y en la práctica de los derechos humanos. Sin embargo ¿es esta estrecha vinculación suficiente para afirmar que la vulnerabilidad constituye el fundamento de los derechos humanos? A través del análisis del contexto histórico en el que surgen los derechos humanos como realidad para el derecho internacional -tras la Segunda Guerra Mundialel autor vuelve a poner en manifiesto la vinculación entre la vulnerabilidad y los derechos humanos, aunque concluye afirmando que el verdadero fundamento de éstos se encuentra en la dignidad humana, así "Vulnerability only becomes relevant for human rights once we have assumed that human being possess intrinsic dignity." (p. 270).

Claudio Sartea es el encargado del Capítulo 12 'The Fragility of the Human Being and the 'Right' to Die: Biojuridical Considerations' en el que analiza el significado y relevancia de la última fase de la vida humana a través de una doble perspectiva: desde la antropología filosófica y desde la filosofía del derecho. En primer lugar considera la mortalidad y la fragilidad como condiciones esenciales de la vida humana, condiciones que relacionará apoyándose en el pensamiento de MacIntyre y Martha Nussbaum con la dependencia como relacionalidad. Estas características humanas son especialmente evidentes en la etapa final de la vida, en las que emergen las reflexiones físicas y psicológicas en torno al sentido de la vida y las relaciones con los demás. Posteriormente realiza una crítica sobre las consideraciones que se encuentran detrás de aquellos pronunciamientos que propugnan por que el derecho se mantenga al margen de estos tópicos, ya que en caso de regular se estaría incurriendo en paternalismo inaceptable. Esta postura será la causante de la gran desorientación en la cultura popular, al igual que la de las decisiones instituciones -tanto en el ámbito legislativo como en el judicial-. Contrario a dicha postura Sartea propone como tesis central la idea de que en la etapa final de la vida el ser humano mantiene la misma dignidad que en cualquier otra etapa toda vez que la mortalidad como se mencionó al principio- es una característica esencial de la condición humana, y es precisamente a través de ésta que los humanos podemos afirmar nuestro carácter de igualdad, quedando en evidencia la ilegitimidad de 
cualquier forma de discriminación por razón de salud o edad. Desde esta perspectiva el autor propone una rehabilitación de los deberes en la etapa final de la vida, deberes que involucran tanto al paciente como a sus familiares y al personal de cuidado. El autor concluye enfatizando la necesidad de reelaborar el concepto de dignidad humana, haciendo frente a reducciones subjetivas e insertándolo en una visión capaz justa e integral tanto de la estructura del ser humano, como de sus relaciones y el marco legal implicado.

\section{El Capítulo 13 'Taking Vulnerability} Seriously: What Does It Change for Bioethics and Politics?' escrito por Corine Pelluchon, resalta la función crítica y política del concepto de vulnerabilidad. Para ello realiza un recorrido y revalorización de la relación autonomía vulnerabilidad, para posteriormente diferenciar su aproximación -aproximación de capacidades y ética de la justicia- de la postura de la ética del cuidado. El valor que tiene la visión que propone queda evidenciado en la siguiente afirmación "Taking into account the vulnerability of the person is to be able to listen to what she says, even when she does not speak.21 This leads us to introduce the main notion of dignity." (p. 300) Posteriormente vinculará el concepto de vulnerabilidad con el de responsabilidad, que no sino otra forma de expresión de la relacionalidad humana, la apertura natural al otro -necesitamos del otro y a la vez estamos involucrados con el destino del otro-, estas consideraciones serán llevadas por Pelluchon al ámbito de la filosofía política, afirmando que el tomar la vulnerabilidad en serio significa en primer instancia tener presente nuestra existencia material. A través de esta filosofía de la corporalidad -prosigue la autora con lo que será el corolario de su intervención- se abre el camino a una filosofía del sujeto menos abstracta e individualista que la que se ha tenido presente en la Cultura Occidental, al igual que a la reconstrucción de una ética y una política que nos permitan hacer frente a la crisis ecológica, y nos permitan ver que la crisis más que ser de recursos es una crisis que concierne a nuestra existencia en la tierra y la forma en la que interactuamos con otros seres.

El último capítulo se encuentra a cargo de Vicente Bellver y lleva por título 'The Principle of Respect for Human Vulnerability and Assisted Reproductive Technologies' En este apartado el autor desarrolla una reflexión en torno a dos controversiales Técnicas de Reproducción Asistida-ARTs' por sus siglas en inglés- que son la subrogación comercial internacional y la transferencia mitocondrial. La elección de estas técnicas no es casual sino que encuentra sentido debido a que ambas exigen especial implicación a mujeres diferentes a aquellas que serán legalmente las madres. Tras exponer las implicaciones de ambas técnicas el autor nos 
muestra el panorama de opinión global que se generó en torno a las mismas, mismo que coincide con las tres posturas típicas respecto al surgimiento de una biotecnología controversial: los 'tecno-entusiastas', los 'prudentes' y los 'desconfiados de la tecnología'. Claramente expone las implicaciones de cada uno de las posturas, poniendo de relieve a la par la gran presión existente en los Estados $\mathrm{y}$ en las organizaciones internacionales para definir sus posturas. Evidentemente ambas técnicas implican dilemas relacionados con la bioética y el bioderecho, mismas que Bellver orienta a través de la reflexión sobre el principio de respeto por la vulnerabilidad humana -Declaración de Bioética y Derechos Humanos de la UNESCOcuestionándose con la compatibilidad entre ambas técnicas y éste principio. Tomando en cuenta los riesgos implicados en ambas técnicas de reproducción asistida, al igual que el principio de vulnerabilidad y su desarrollo previo a la inclusión en el artículo 8 de la declaración de la UNESCO, el autor concluye poniendo en duda sobre la conveniencia de regular dichas prácticas, haciendo un llamado a una prohibición universal efectiva. 